\title{
Comparative Effects of Repetitive Odor Identification and Odor Memory Tasks on Olfactory Engagement in Older Populations - A Pilot fMRI Study
}

\author{
Narayan Rai ${ }^{1} *$ \\ Maria Mananita Hipolito I,* \\ John W VanMeter ${ }^{2}$ \\ Riya Seth ${ }^{3}$ \\ Ayokunnumi Adenuga ${ }^{3}$ \\ Myeshia Shelby ${ }^{4}$ \\ Magdalena Misiak-Christian ${ }^{5}$ \\ Charles Nwaokobia (D) $^{3}$ \\ Kebreten F Manaye ${ }^{5}$ \\ Thomas O Obisesan $\mathbb{1 D}^{6}$ \\ Evaristus Nwulia $\mathbb{D}^{1,3}$ \\ 'Department of Psychiatry and \\ Behavioral Sciences, Howard University, \\ Washington DC, USA; ${ }^{2}$ Department of \\ Neurology, Center for Functional and \\ Molecular Imaging, Georgetown \\ University Medical Center, Washington \\ DC, USA; ${ }^{3}$ Evon Medics LLC, Elkridge, \\ MD, USA; ${ }^{4}$ Howard University Graduate \\ School, Washington DC, USA; \\ ${ }^{5}$ Department of Physiology, Howard \\ University, Washington DC, USA; \\ ${ }^{6}$ Department of Medicine, Howard \\ University, Washington DC, USA \\ *These authors contributed equally to \\ this work
}

Correspondence: Evaristus Nwulia Department of Psychiatry and Behavioral Sciences, Howard University, Washington DC, WA, USA

Email enwulia@howard.edu
Objective: This study evaluated human Blood Oxygen Level-Dependent (BOLD) responses in primary and higher-order olfactory regions of older adults, using odor memory and odor identification tasks. The goal was to determine which olfactory and memory regions of interest are more strongly engaged in older populations comparing these two odor training tasks.

Methods: Twelve adults $55-75$ years old (75\% females) without intranasal or major neurological disorders performed repetitive odor memory and identification tasks using a 3-tesla magnetic resonance scanner. Odors were presented intermittently at 10 -second bursts separated by 20 -second intervals of odorless air. Paired $t$-tests were used to compare differences in the degree of activation between odor identification and odor memory tasks within individuals. An FDR cluster-level correction of $p<0.05$ was used for multiplicity of tests (with a cluster-defining threshold set at $p<0.01$ and 10 voxels).

Results: Odor identification compared to memory (ie, odor identification $>$ odor memory) contrasts had several areas of significant activation, including many of the classical olfactory brain regions as well as the hippocampus. The opposite contrast (odor memory > odor identification) included the piriform cortex, though this was not significant. Both tasks equally activated the piriform cortex, and thus when the two tasks are compared to each other this area of activation appears to be either absent $(\mathrm{OI}>\mathrm{OM})$ or only weakly observed $(\mathrm{OM}>\mathrm{OI})$.

Conclusion: These findings from a predominantly African American sample suggest that odor identification tasks may be more potent than memory tasks in targeted olfactory engagement in older populations. Furthermore, repetitive odor identification significantly engaged the hippocampus - a region relevant to Alzheimer's disease - more significantly than did the odor memory task. If validated in larger studies, this result could have important implications in the design of olfactory training paradigms.

Keywords: blood oxygen level dependent responses, BOLD, odor memory, odor identification, olfactory engagement, olfactory training

\section{Background}

Functional neuroimaging procedures have contributed immensely to improved understanding of the neurobiology of olfactory processing and to the elucidation of the effects of normal and abnormal aging on the risks of Alzheimer's disease development. $^{1,2}$ Functional magnetic resonance imaging (fMRI), measuring the Blood Oxygen Level Dependent (BOLD) response to neural activation in the olfactory cortex, has particularly enabled the identification of cortical and subcortical brain structures that participate in olfactory processing. ${ }^{3,4}$ Examining the sense 
of smell with olfactory fMRI is a sensitive technique ${ }^{5}$ to demonstrate neural correlates of altered performance in a number of olfactory tasks and can be used to detect selective activation of the primary and higher-order olfactory region by olfactory psychophysical tasks. There is increasing evidence from clinical studies that olfactory training, by repetitive odor stimulation with or without odor psychophysical tasks, can improve functional deficits in olfactory neural structures, which are impacted early by Alzheimer's disease pathophysiology. ${ }^{6-8}$ To provide empirical support for the use of a particular olfactory psychophysical task over others, as a putative tool for treatment or prevention of age-dependent olfactory compromise, it is very important to compare psychophysical tasks on their relative strengths for functional engagement of these regions of interest.

Psychophysical tasks of olfaction are based upon the presentation of odors to a test subject, followed by examination of the subject's responses to questions about some characteristics of the odorants presented. ${ }^{9,10}$ Olfactory psychophysical tasks include odor identification, odor memory, odor discrimination and odor threshold sensitivity tasks. Odor identification (OI) is the number of odorants a participant can correctly identify or name out of the total number of odorants presented. Odor memory (OM) measures the ability to remember recently administered odorants from a choice that includes non-previously administered odors. Odor threshold (OT) measures the lowest concentration of an odorant that can be reliably perceived. Odor discrimination measures ability to differentiate between two alternately administered odors. Odor memory task is considered to pose the highest cognitive demands, and it has been shown to extensively activate olfactory brain regions like piriform and orbitofrontal cortices. ${ }^{11}$ The piriform cortex is the most distinct and the largest part of the primary olfactory cortex. It is interconnected with higher-order olfactory regions and other primary olfactory regions. ${ }^{12-14}$ fMRI studies have shown that the primary olfactory cortex was activated inconsistently or not at all during passive olfactory stimulation, whereas the higher-order olfactory region showed strong activation. ${ }^{15-17}$ Poellinger et a ${ }^{18}$ demonstrated from timeseries fMRI experiments in adult populations that faster habituation to odor stimuli in the primary olfactory cortex contributed to the inconsistencies by which odor stimuli activated the piriform and other primary olfactory cortex regions. Specifically, they showed that the primary olfactory cortex habituates after 10 seconds of continuous odor delivery in the general adult population. It therefore follows that odor presentations during odor processing tasks that depend on structure and function of the piriform cortex (eg, odor memory) must be brief.

The purpose of this study is to examine the relative differences in olfactory engagement comparing the two odor training tasks of memory and identification in older populations using an olfactory fMRI paradigm in twelve individuals without neurological and nasal disorders. These tasks were used to identify the selective activation of the primary olfactory cortex and higher-order olfactory regions. Intermittent odor delivery with short duration bursts was used to minimize habituation to the odorants used in this study. Results from this study are anticipated to have some implications in the design of olfactory fMRI scanning paradigms that can be used in future studies of olfaction in older populations, and for future developments in olfactory training paradigms in this population.

\section{Methods}

\section{Participants}

Twelve adults, 55-75 years of age, were recruited via the geriatric clinic at Howard University and from communities in Wards 7 and 8 of Washington, DC, a population that is predominantly of African American (AA) ethnicity. Study procedures were approved by the Howard University Institutional Review Board (IRB) for the ethical conduct of research involving human subjects in accordance with the Declaration of Helsinki. Only volunteers that provided written informed consent to participate following a complete discussion of the study were enrolled into the study. To be eligible for this study, participants must have little to no memory impairments, no history of psychiatric disorder (based on detailed psychiatric history and exam performed by the neuropsychiatrist co-author, EN), no current substance use (determined from urine drug toxicology screen and blood alcohol levels), no anosmia based on odor threshold sensitivity test scores, absence of cerebrovascular disease or other neurologic disease (eg multiple sclerosis, Parkinson's disease, Lewy body dementia, frontotemporal dementia or traumatic brain injury), no significant nasal disorders (eg nasal polyps or nasal tumors, deviated septum, nasal surgeries, congenital malformations of the nose, chronic nasal obstruction and chronic rhinosinusitis), and no serious medical disorders (eg end-stage kidney disease and congestive heart failure). For exclusion purposes, neurological diseases were ascertained through detailed neurological exam by the 
neuropsychiatrist co-author $(\mathrm{EN})$, medical records review and from structural MRI. Structural MRI was reviewed by the neuroradiology co-author (JWV), and people with structural brain lesions were excluded. In addition to self-report and medical records review, all participants received comprehensive nasal inspection using flexible nasoscope. We also conducted olfactory psychophysical tasks using a validated olfactometer (described below), to exclude people with reduced odor threshold (OT) sensitivity, defined as score $<6$ points out of a maximum score of 10 points. Detailed social, demographic and medical history were obtained from all participants. Mini-Mental Status Examination (MMSE) was conducted on all participants to exclude people with Alzheimer's dementia or mild cognitive impairments (MCI). Only subjects with MMSE scores $>27$ out of a maximum of 30 were included in this study. Buccal swabs were collected from each participant to genotype for apolipoprotein E (APOE) polymorphisms. All subjects were screened before scanning to ensure compatibility with a magnetic resonance (MR) environment; as such, to be included in this study, subjects were not claustrophobic, had no metallic implants and were able to remove all metallic or magnetic devices, if present. Additionally, on the day of MRI scanning all participants completed detailed MRI safety form to identify and exclude people with MRI exclusions.

\section{Olfactory Data Collection}

\section{Psychophysical Tasks of Olfaction}

This was conducted using the computer-controlled OLFACT-Combo, a flow-dilution olfactometer, ${ }^{19}$ in a quiet room by research staff. This olfactometer delivers various odorants intranasally for clinical assessment of odor identification (OI), odor memory (OM), odor threshold (OT) and odor discrimination (OD). ${ }^{8}$ OLFACT consists of a two-chambered metal box; one chamber, which is used for OT task, contains 13 vials of n-butanol solution in different concentrations and an empty vial for clean air; and the other chamber contains 20 different vials of essential oils and is used for OI, OM and OD tasks. Details of olfactory evaluation by means of OLFACT-COMBO has been described elsewhere. ${ }^{20}$ To prevent crosscontamination of odorants, a Teflon tube is attached to each vial and presented through a plastic tube. Participants sat comfortably in front of a laptop connected to the olfactometer, for presentation of the olfactory task questions. Test instructions and a practice trial were provided before each olfactory task assessment. The Osmic software installed on the laptop collected and stored participants' responses. Test-retest reliabilities of OLFACT for OI, OM, OT and OD were 0.86, 0.84, 0.77 and 0.79 , respectively. ${ }^{21}$ Only normosmic participants, ie, individuals that scored 6 or more points out of a maximum score of 10 in the odor sensitivity task, were included in the study.

\section{Odor Identification and Memory fMRI Olfactory Paradigm}

The fMRI olfactory paradigm employed a portable laptopcontrolled MRI-compatible olfactometer, called OLFACTfMRI. $^{22}$ OLFACT-fMRI consists of an air delivery system contained in a metal box and an odorant delivery system in a plastic box container. The laptop and metal box were placed outside the MRI scanner room, and a detachable non-metal delivery hose $(24 \mathrm{ft} \times 1.25 \mathrm{in})$ connects the metal box with the MRI-compatible plastic box, through an open waveguide. The odorant delivery system, which contains six different essential oils, was placed alongside the study participant. Odorants were delivered from the plastic box using a plastic nasal cannula modified with a Y-shaped Teflon tube at its tip and placed into the subject's nostrils. The Teflon tube is coated with polytetrafluoroethylene (PTFE), a synthetic chemical that provides a nonreactive, nonstick and almost frictionless surface. ${ }^{23}$ A PTFE-coated tube, unlike a plastic tube, is extremely chemically resistant, avoids adhesion and enables unrestricted flow without deposit build-up. To hold the Teflon tube in place, the plastic tubing is placed over the ear and behind the head, ensuring the comfort of the study participant and the reliable delivery of the odorants. Odor presentation was triggered by the OLFACT software. A constant stream of clean odorless (maximum flow rate of up to 2 liters per minute), non-heated and nonhumidified air was delivered in between odorant delivery. The highest concentration of the odorants was used for the olfactory tasks; and the essential oils were purchased from Save on Scents and Perfumer's Apprentice.

The acquisition (ie, odor encoding) stage of the OM task from the fMRI olfactory paradigm started in the mock scanner room. Participants were instructed to breathe normally and to remember the odorants presented while in the mock scanner. Each participant was asked to smell three essential oils (lemon, cinnamon and rose) sequentially for 3 cycles; odor was presented for 10 seconds followed by a 20-second gap to control for odor adaptation. The total duration of the acquisition stage was 280 seconds. Participants were instructed that a screen inside the MRI 
scanner will ask them whether the odor was one of the three they acquired during the acquisition stage; all subjects practiced using the response box.

During the recognition stage of the OM task, conducted in the MRI scanner fifteen minutes after the acquisition stage, participants were presented six odorants (strawberry, rose, coconut, lemon, cherry and cinnamon). During the 10second presentation of an odor, the screen displayed the query as to whether they remembered the odor from the acquisition phase. This was followed by 20 seconds of no odor presentation, during which subjects were instructed to breathe normally. The series of six odorants were presented three times in identical order, for eighteen total odor presentations. Subjects were instructed to press the button in their right hand for recollection or the button in their left hand for non-recollection (Figure 1A). The response was limited to 10 seconds of odor exposure, not during the 20second rest intervals. Ten seconds of rest/no odor was included at the beginning and end of the task. The total duration of the task was 560 seconds.

The entire fMRI OI task was conducted in the scanner room. In the scanner, the subject held a three-button response box in each hand - one button each for the index, middle and ring finger of each hand, with each finger corresponding to the image of the odorant shown on the screen during odor presentation, interspersed by twenty seconds of breathing normally (Figure 1B). Six essential oils (strawberry, rose, coconut, lemon, cherry and cinnamon) were presented sequentially for 3 cycles, and each odorant was presented for 10 seconds, followed by a 20 -second gap in between. Subjects were instructed to press the button corresponding to the on-screen image of the scent sensed. For instance, while the strawberry scent is being inhaled, selection of the on-screen image of strawberry, among other scent images to select from, is the correct OI response; and selecting the image of a different scent instead of strawberry image is the wrong response. Participants were asked to make a choice within each 10 seconds of odor exposure. Ten seconds of rest/no odor was included at the beginning and end of the task. The total duration of the task was 560 seconds.

\section{MR Acquisition}

Participants were scanned using functional MRI, that was acquired on a Siemens 3T Prisma-Fit MRI scanner with a 64channel head coil using an echo-planar imaging pulse sequence with $\mathrm{TR} / \mathrm{TE}=859 / 35 \mathrm{~ms}$, multiband factor of 6 , FOV $=200 \mathrm{~mm}^{2}$ and a $100 \times 100$ matrix, and 60 2-mm thick slices for an effective spatial resolution of $2 \mathrm{~mm}^{3}$. For localization and spatial normalization, a high-resolution T1-weighted
A

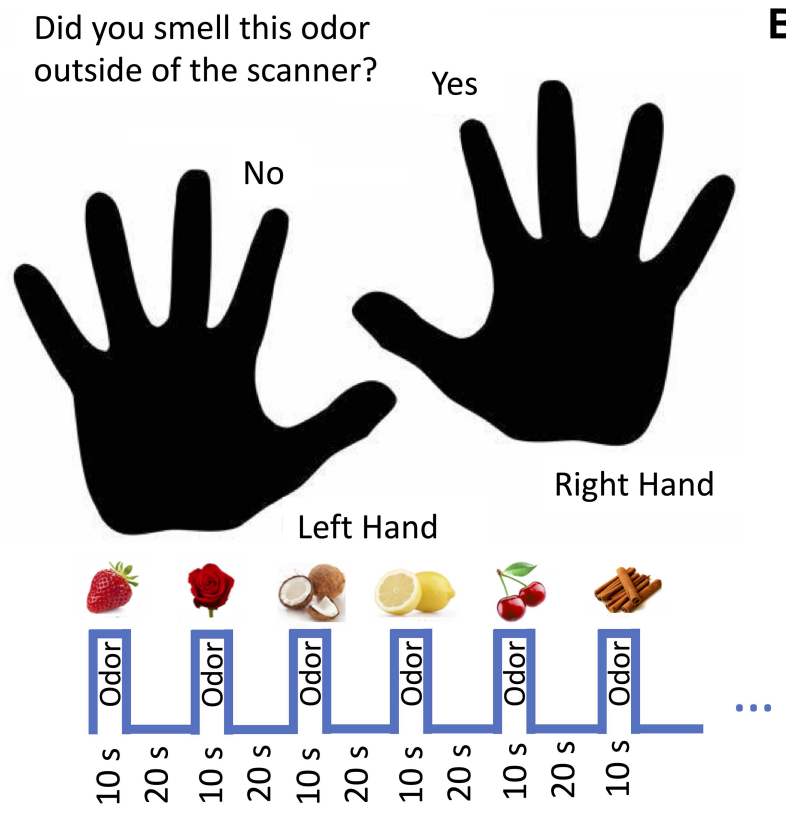

Cycle Repeated Three Times
B Which odor did you smell?

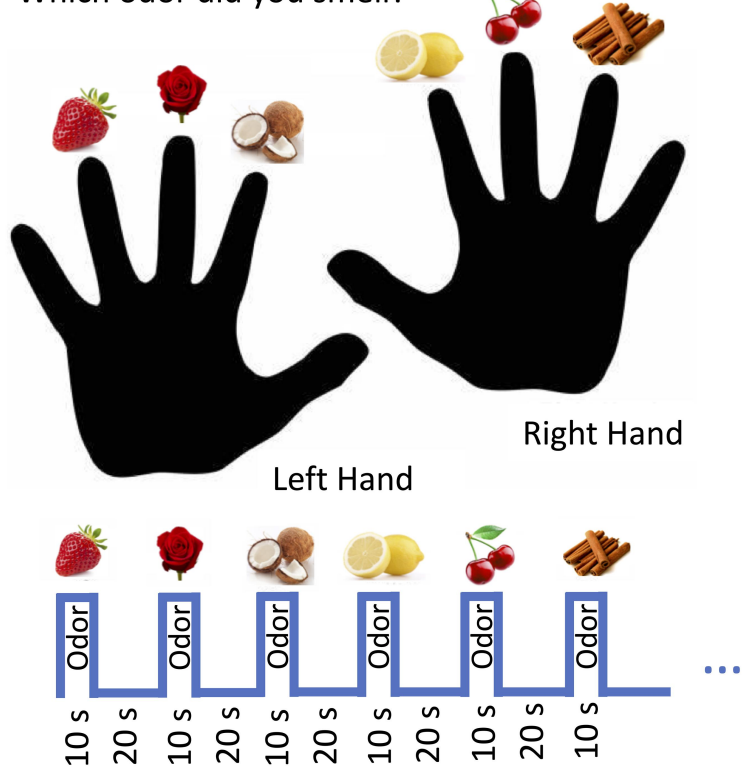

Cycle Repeated Three Times

Figure I Experimental design of the odor identification and odor memory fMRI tasks. (A) Odorants were first presented outside the scanner, followed by I0-second presentations of those odors and novel odors with questions asking subjects to indicate with their fingers if they recognized the old odors. Odor presentations are interspersed by 20 -second rest intervals of odorless air flow. (B) Six odorants were presented one at a time for 10 seconds, interspersed by 20 -second odorless intervals. Participants were instructed to use their fingers to point to the name of odorants on the screen corresponding to the smell they were perceiving. 
MPRAGE scan was acquired with TR/TE/TI = 1900/2.9/900 $\mathrm{ms}, \mathrm{FOV}=256 \mathrm{~mm}^{2}$ and a $256 \times 256$ matrix, 176 slices for a $1 \mathrm{~mm}^{3}$ spatial resolution.

\section{Analysis}

Data were analyzed in SPM12 (Wellcome Trust Centre for Neuroimaging, University College London), and analysis consisted of the following steps: slice timing correction to temporally align slices, realignment to correct for head motion, co-registration of the mean functional scan to the high-resolution structural scan, spatial normalization of the high-resolution structural scan that was subsequently applied to the realigned functional scans and smoothing with a $4 \mathrm{~mm}^{3}$ filter.

Further analysis consisted of conducting a repeated measures 1-way ANOVA combining first-level contrast maps for odor versus no-odor using both paradigms. Age was entered as a covariate of no interest. A cluster defining uncorrected threshold of $p<0.001$ and 10 contiguous voxels was used followed by use of a cluster-level FDRcorrected $p$-value of 0.05 .

\section{Results}

\section{Participants' Characteristics}

The study population consists of 12 total individuals, 9 $(75 \%)$ of whom are females, and predominantly of African American ethnicity (Table 1). Socio-demographic features and performance in fMRI olfactory tasks of the study population are also depicted in Table 1. Participants correctly identified $17.9 \%$ of the odorants presented to them during odor identification fMRI task, with percent identifications ranging from $0 \%$ to $39 \%$. During fMRI scanning, the study population correctly recognized 62\% (range $39-78 \%$ ) of odorants they were previously exposed to during the acquisition stage.

\section{fMRI Outcomes}

Results of comparison of odor identification (OI) $>$ odor memory (OM) showed statistically greater activation of several olfactory brain regions, including right middle temporal, bilateral precuneus, right hippocampus, left superior parietal, right superior parietal, left middle temporal, left inferior orbital frontal, left superior frontal, left insula and bilateral cerebellum (culmen and vermis) (Table 2, Figure 2).

By comparison, the odor memory task did not exhibit any areas with significantly greater activation than odor
Table I Socio-Demographic Characteristics and Olfactory Task Performances in the Study Population

\begin{tabular}{|c|c|c|}
\hline Characteristics & $\begin{array}{l}\text { Frequency } \\
\qquad(n=12)\end{array}$ & Percentage \\
\hline Female & 9 & 75 \\
\hline \multicolumn{3}{|l|}{ Race: } \\
\hline Black/African American & 11 & 99.67 \\
\hline Other & I & 8.33 \\
\hline \multicolumn{3}{|l|}{ Education: } \\
\hline $8-12$ years & 5 & 41.67 \\
\hline Graduate/Some college/Associate & 7 & 58.33 \\
\hline \multicolumn{3}{|l|}{ Employment status: } \\
\hline Retired & 7 & 58.33 \\
\hline Self-employed & I & 8.33 \\
\hline Unemployed & 4 & 33.33 \\
\hline \multirow[t]{2}{*}{ Never married } & 5 & 41.67 \\
\hline & Mean (SD) & Range \\
\hline Age & $66.08(4.99)$ & $55-74$ \\
\hline fMRI Tasks of Olfaction & Mean (SE) & Range \\
\hline Odor Identification & $17.9 \%(3.3 \%)$ & $0-39 \%$ \\
\hline Odor Memory & $62.0 \%(3.9 \%)$ & $39-78 \%$ \\
\hline
\end{tabular}

identification task. The right and left piriform cortices were slightly more activated for OM than OI, though these did not reach statistical significance (Table 3, Figure 3). Examination of each of the tasks in isolation using one-sample $t$-tests revealed that neither task robustly activated the piriform cortex.

Finally, it is important to note that both tasks activated the piriform cortex, and thus when the two tasks were compared to each other this area of activation appears to be either absent (OI > OM) or only weakly observed (OM $>$ OI). In Supplementary Table 1 (see Supplementary docu ments) we present a one-way ANOVA using repeated measures to combine the data from both tasks to demonstrate that this part of the olfactory sensory cortex is activated by these tasks. A cluster-determining threshold of $p<0.001$ uncorrected and a cluster extent of 10 voxels were used.

\section{Discussion}

Olfactory training, comprising of repetitive daily stimulation with odorants, has been proposed as putative treatment for several local and intracranial diseases in which olfactory functions are compromised. ${ }^{7,24,25}$ In this present study we 
Table 2 Results of Comparison of Odor Identification (OI) and Odor Memory (OM) Tasks (ie, OI > OM Contrasts), Depicting Olfactory Brain Regions Which Were More Significantly Activated by OI Compared to OM Tasks

\begin{tabular}{|c|c|c|c|c|}
\hline Brain Region & Peak T Statistic & FDR Cluster Corrected $p$-value & Cluster Size & MNI Coordinates \\
\hline R Middle Temporal & 12.30 & 0.000 & 1306 & $56,-48,0$ \\
Bilateral Precuneus & 5.89 & & $4,-56,44$ \\
R Hippocampus & 4.47 & & $40,-38,-8$ \\
L Superior Parietal & 5.50 & & $-24,-72,50$ \\
R Superior Parietal & 5.14 & & $30,-72,46$ \\
L Middle Temporal & 3.34 & 0.000 & $-42,-52,-4$ \\
\hline L Inferior Orbital Frontal & 5.95 & & $-32,36,-16$ \\
L Superior Frontal & 5.22 & & $-12,46,18$ \\
L Insula & 3.45 & $0.087^{*}$ & $-26,20,-18$ \\
\hline R Medial Orbital Frontal & 5.36 & $0.055^{*}$ & $8,52,-16$ \\
\hline L Medial Orbital Frontal & 4.54 & & 257 & $-12,42,-10$ \\
L Caudate & 3.80 & 4.67 & $0.055^{*}$ & $-18,22,0$ \\
\hline R Superior Parietal & 4.18 & 0.014 & 261 & $24,-36,70$ \\
\hline Bilateral Cerebellum (Culmen and Vermis) & & & 392 \\
\hline
\end{tabular}

Note: *Regions that are included even though they are not significant following FDR correction as these areas are involved in olfaction, and with more subjects would have reached significance.

examined human BOLD activities in primary olfactory cortex and higher-order olfactory regions in response to repetitive olfactory cognitive tasks in older individuals, to determine their potential relevance to olfactory training for age-associated neurological conditions. The comparisons between odor identification activations and odor memory activations were made within an individual level, since every participant received both tasks at different time points. Therefore, individuals serve as controls in contrast to grouplevel studies where some individuals were randomly assigned just one or the other task. Within-individual analysis helps to reduce the effects of heterogeneity introduced by differences between individuals. In older adults without nasal and neurological disorder, the odor identification (OI) task significantly activated several brain regions more than did the odor memory (OM) task, including the right middle temporal, bilateral precuneus, right hippocampus, left superior parietal, right superior parietal, left middle temporal, left inferior orbital frontal, left superior frontal, left insula and bilateral cerebellum (culmen and vermis). The OM task, in contrast, activated the piriform cortex more than did the OI task, though it was not significant. Both tasks equally activated the piriform, and thus when the two tasks were compared to each other, within individuals, this area of activation appears to be either absent (OI > OM) or only weakly observed (OM > OI). However, OI did preferentially activate the hippocampus in comparison to the OM task. This study provides new evidence that the OI task, when used for olfactory training in older subjects, engages several olfactory regions and the hippocampus, as compared to the OM task.

Though several studies have been conducted on patterns of brain activations in the general population, fewer studies have examined changes in these patterns of activation in elderly populations in efforts to elucidate the impact of aging on the human olfactory system. This study in older populations revealed that odor cognitive fMRI tasks activated most of the classic primary and secondary olfactory areas activated by odorants in the general population, including the hippocampus, insula, inferior orbital frontal, medial orbital frontal, middle temporal, precuneus and parietal regions. ${ }^{18,26-28}$ Consistent with a previous study by Kareken et al, ${ }^{29}$ OI activated temporal lobes, orbitofrontal cortices, right hippocampus ${ }^{27}$ and left insula areas responsible for higher-order mental processing. ${ }^{30}$ It is noteworthy that at least one $\operatorname{study}^{26}$ did not find significant activations in any of these classical olfactory brain regions in elderly subjects, during fMRI odor identification tasks. One possible explanation for absence of olfactory regional activations in the latter study is their much older age cohort: the mean (SD) age of participants in their study is 73 (5) years, compared to 66 (5) years in our study. Other contributing factors could be differences in methodology, such as 


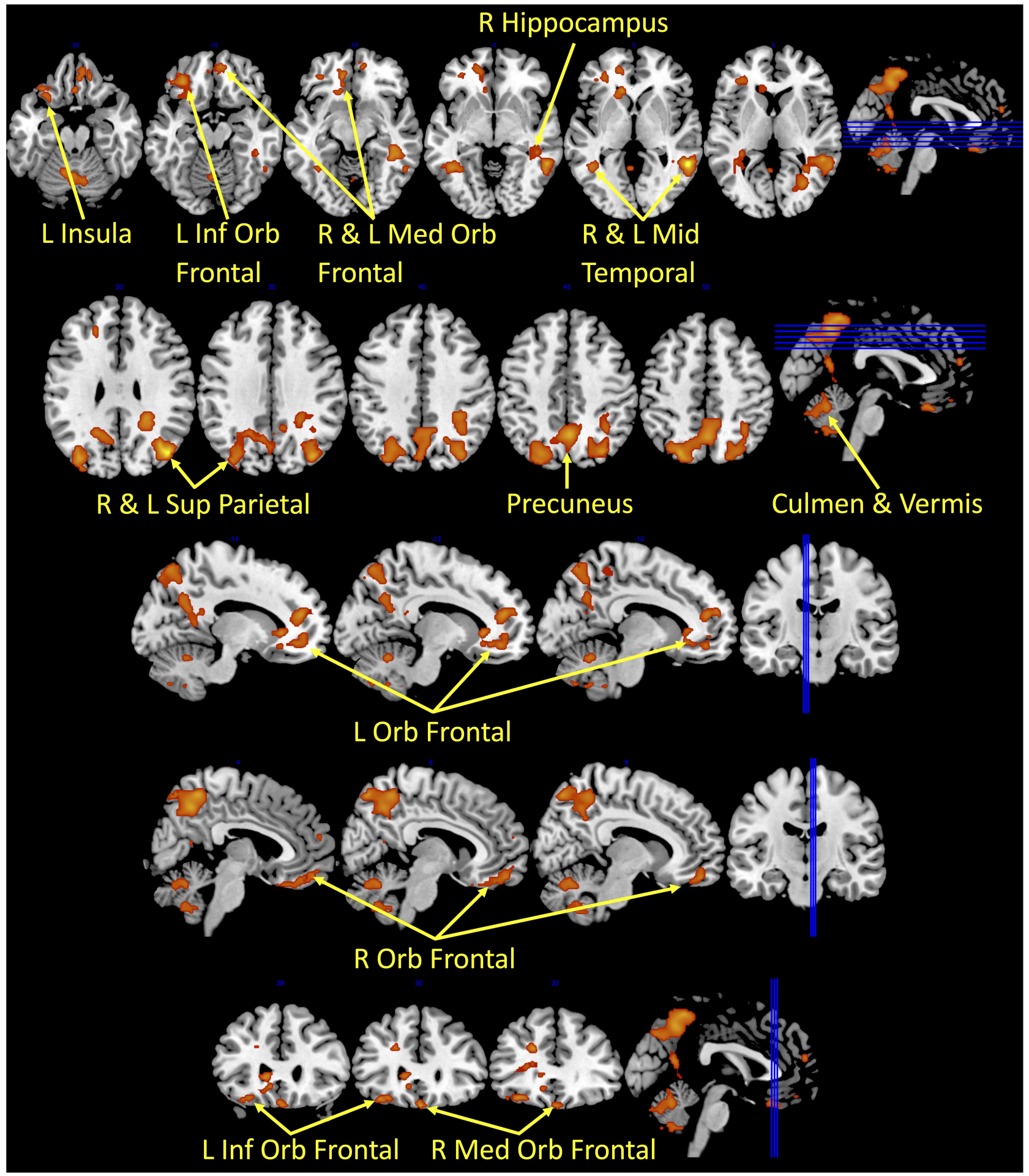

Figure 2 Olfactory brain regions exhibiting greater activation for odor identification task than for odor memory task contrast.

use of different odors and their use of longer rest intervals between odor stimuli.

In contrast to broad activations of odor identification tasks in both primary and secondary olfactory regions, odor memory (OM) tasks have been found to activate mostly the primary olfactory cortex, particularly the piriform cortex. ${ }^{31}$ To the best of our knowledge, there are no published studies specifically investigating regional differences in brain activations following odor memory fMRI tasks in elderly versus younger subjects. We 
Table 3 Result of Analysis of Piriform Cortex Activation for the Odor Memory (OM) Task Greater Than Odor Identification Task (ie, OM > OI) Contrast. Greater Activation of the Piriform Cortex by OM > OI Contrast Was Not Statistically Significant

\begin{tabular}{|l|c|c|c|c|}
\hline Brain Region & Peak T Statistic & FDR Cluster Corrected p-value & Cluster Size & MNI Coordinates \\
\hline R Piriform & 2.57 & 0.944 & 83 & $16,0,-6$ \\
L Piriform & 2.61 & 0.944 & 77 & $-20,-2,-8$ \\
\hline
\end{tabular}

anticipated that OM tasks would activate the piriform cortex more significantly than would the OI task, but as we showed in the Supplementary data, both tasks equally activated the piriform cortex to a great extent. One possible reason why the piriform activation by the $\mathrm{OM}$ task did not significantly exceed piriform activation by the OI task is that age-associated biological factors could have imposed limitations to the extent of piriform activation by memory tasks. Alternatively, especially given that there was a trend toward greater piriform activation by the OM versus the OI task, it is possible that the superiority of OM-based piriform activation over OIbased piriform activation would be more statistically significant in larger clinical samples. Finally, it is also conceivable that the aging piriform cortex habituates even more quickly than in the general population. We used very brief, 10-second burst stimulation sequences, which Poelinger et al used to successfully activate the piriform cortex in the general population. In further support of our use of 10 -second stimuli, we showed significant activation of the piriform by the OM task (Supplementary Table 1), and the only problem here is that OI equally activated the piriform in these participants.

This study also has implications for olfactory training using repetitive odor cognitive tasks in older adults. From this this perspective, repetitive presentations of odor identification tasks produce stronger engagement of primary and secondary olfactory cortex regions, and the hippocampus, compared to repetitive presentations of odor memory tasks in this population. An important question that arises is why the odor memory greater than odor identification (ie, OM > OI) contrast did not reveal significant differences in activation of nearby medial temporal regions, such as parahippocampus and hippocampus, which are usually engaged by odor memory tasks in younger cohorts. A possible explanation can be drawn from previous discoveries that secondary and higher-order central olfactory structures, which contribute to odor identification, showed significant agedependency during middle age and early part of aging, while the primary olfactory cortices - essential to episodic odor memory - did not show significant agedependency in these age groups. ${ }^{32}$ This is further supported by evidence of selective atrophy of secondary olfactory structures (eg, orbitofrontal cortex), but not the primary olfactory cortex during normal aging, ${ }^{33}$ and by our finding of relatively poor task performance for odor identification (ie, OI performance score) in comparison to odor memory (OM performance score), reported in Table 1. Several studies support the notion that odor cognitive or training tasks which target regions more strongly impacted by age-related atrophy are more likely to elicit functional changes, compared to tasks that target regions that are relatively spared. ${ }^{32}$ This could partly explain why passive odor exposure strongly activated the piriform cortex in our study population, whereas the odor memory task was unable to do so.

This study can be viewed in the context of its numerous strengths and limitations. The sample size was small, which might have impacted the odor memory task and the lack of significant difference in primary olfactory cortex. This also limits our ability to conduct sub-group analysis, including sex- and ApoE genotype-specific effects. Another limitation is the possible effect of habituation on identification of primary cortex areas during odor cognitive fMRI paradigms. Our paradigm consisted of 10 -second-long presentations of odorants separated by 20 -second rest intervals. A previous study investigating regional patterns of activations and habituations suggest that primary olfactory regions habituate faster, usually after 10 seconds. ${ }^{18}$ Although the 10 -second duration used in our study is within the window of time before desensitization or habituation in the latter study, it is possible that the 20 -second rest intervals led to both shorter neural recovery times and greater signal drop-off in older individuals. Despite these limitations, this study makes a considerable advancement to the field by studying older US populations of African ancestry, who are often underrepresented in biomedical and neuroscience studies, but who, at the same time, suffer higher prevalence of severe neurodegenerative diseases. 


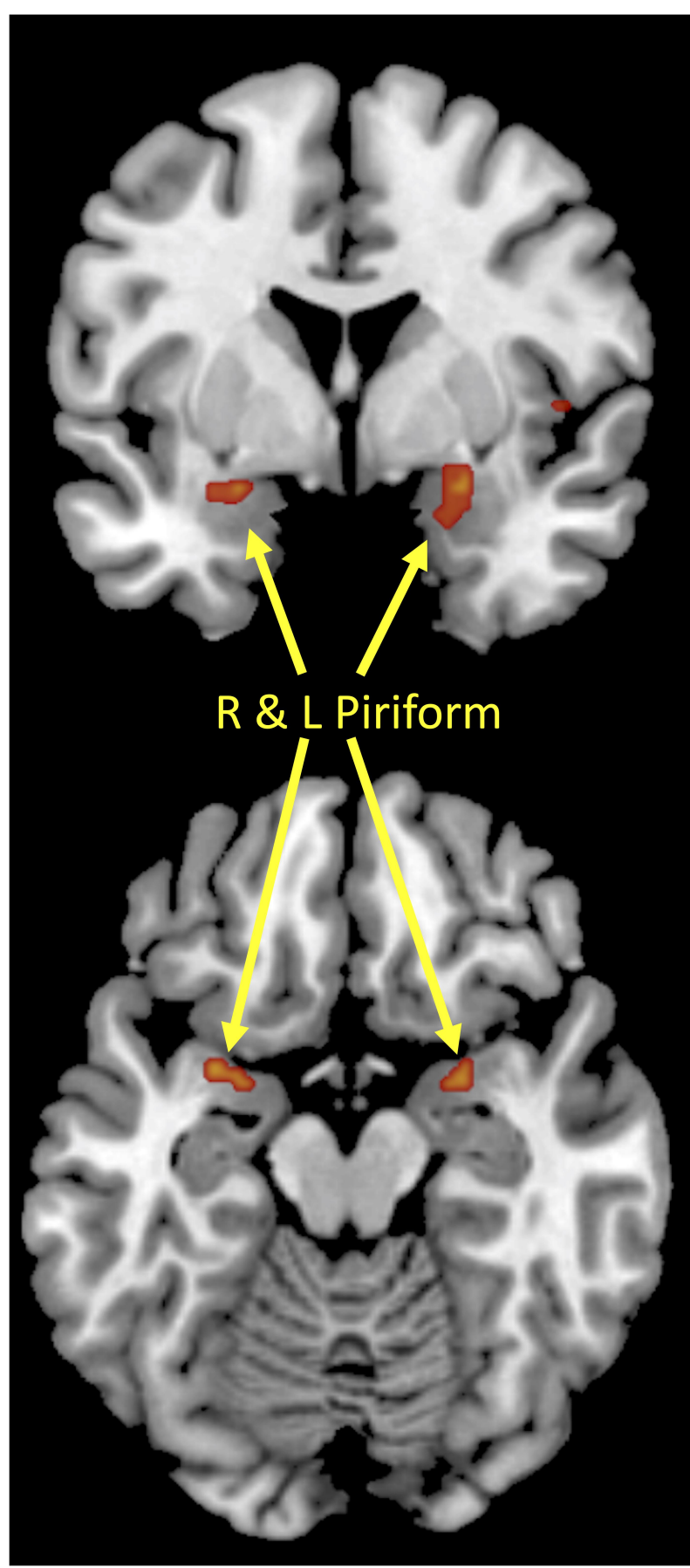

Figure 3 Activation of right and left piriform cortices by both odor memory and odor identification tasks compared to no odor contrast. Odor memory slightly activated the piriform more than the odor identification, but this greater activation $(\mathrm{OM}<\mathrm{OI})$ was not statistically significant.

\section{Conclusion}

This investigation of BOLD signal responses to repetitive OI versus OM tasks shows that OI more potently engaged several olfactory brain regions, compared to OM tasks. Although both tasks activated the piriform cortex, no task was superior to the other in piriform activation.
The OI task, however, did significantly engage the hippocampus - a region relevant to Alzheimer's disease - more significantly than did the OM task. Results from this study have important implications on the design of olfactory fMRI scanning paradigms that can be used in future studies of olfaction in older populations. Future studies in larger samples are needed to investigate whether important biological factors, such as ApoE genetic variations and neurodegenerative changes, contribute to heterogeneity of the effects of repetitive odor cognitive tasks on brain functions.

\section{Abbreviations}

BOLD, Blood Oxygen Level Dependent; fMRI, functional magnetic resonance imaging; MCI, mild cognitive impairments; MMSE, Mini-Mental State Examination; MR, magnetic resonance; OD, odor discrimination; OI, odor identification; OM, odor memory; OT, odor threshold; PTFE, polytetrafluoroethylene.

\section{Acknowledgment}

We would like to thank our otolaryngologist, Dr. Adedoyin Kalejaiye, for her ear nose and throat (ENT) advice and consultations for this study.

\section{Funding}

This study is supported by USPHS grants R43AG061981 (S.S. and E.N.) and R01AG063881 (E.N., M.M. and T.O).

\section{Disclosure}

$\mathrm{Mr}$ Charles Nwaokobia reports grants from National Institute on Aging, during the conduct of the study; in addition, Mr Charles Nwaokobia has a patent 10016473 issued; Dr Evaristus Nwulia reports grants from Evon Medics LLC, during the conduct of the study. The authors report no other conflicts of interest in this work.

\section{References}

1. Zhou G, Lane G, Cooper SL, Kahnt T, Zelano C. Characterizing functional pathways of the human olfactory system. ELife. 2019;8. doi:10.7554/eLife.47177.

2. Toepper M. Dissociating normal aging from Alzheimer's disease: a view from cognitive neuroscience. $J$ Alzheimers Dis. 2017;57:331-352. doi:10.3233/JAD-161099

3. Li W, Howard JD, Parrish TB, Gottfried JA. Aversive learning enhances perceptual and cortical discrimination of indiscriminable odor cues. Science. 2008;319:1842-1845. doi:10.1126/ science. 1152837

4. Karunanayaka P, Eslinger PJ, Wang J-L, et al. Networks involved in olfaction and their dynamics using independent component analysis and unified structural equation modeling. Hum Brain Mapp. 2014;35:2055-2072. doi:10.1002/hbm.22312 
5. Wang J, Eslinger PJ, Doty RL, et al. Olfactory deficit detected by fMRI in early Alzheimer's disease. Brain Res. 2010;1357:184-194. doi:10.1016/j.brainres.2010.08.018

6. Kollndorfer K, Kowalczyk K, Hoche E, et al. Recovery of olfactory function induces neuroplasticity effects in patients with smell loss. Neural Plast. 2014;2014:1-7. doi:10.1155/2014/140419

7. Schriever VA, Lehmann S, Prange J, Hummel T. Preventing olfactory deterioration: olfactory training may be of help in older people. $J \mathrm{Am}$ Geriatr Soc. 2014;62:384-386. doi:10.1111/jgs.12669

8. Doty RL. Olfaction. Ann Rev Psychol. 2001;52:423-452. doi:10.1146/annurev.psych.52.1.423

9. Misiak MM, Hipolito MS, Ressom HW, Obisesan TO, Manaye KF, Nwulia EA. Apo E4 alleles and impaired olfaction as predictors of Alzheimer's disease. Clin Exp Psychol. 2017;03. doi:10.4172/24712701.1000169 .

10. Doty RL. Olfactory capacities in aging and Alzheimer's disease ${ }^{\mathrm{a}}$. Ann N Y Acad Sci. 1991;640:20-27. doi:10.1111/j.1749-6632.1991. tb00185.x

11. Savic I, Gulyas B, Larsson M, Roland P. Olfactory functions are mediated by parallel and hierarchical processing. Neuron. 2000;26:735-745. doi:10.1016/S0896-6273(00)81209-X

12. Carmichael ST, Clugnet M-C, Price JL. Central olfactory connections in the macaque monkey. J Comp Neurol. 1994;346:403-434. doi:10.1002/cne. 903460306

13. Price JL. Olfactory System. San Diego: Academic Press; 1990.

14. Shipley MT, Ennis M. Functional organization of olfactory system. $J$ Neurobiol. 1996;30. doi:10.1002/(SICI)1097-4695(199605) 30:1<123::AID-NEU11 $>3.0 . \mathrm{CO} ; 2-\mathrm{N}$.

15. Wilson DA. Habituation of odor responses in the rat anterior piriform cortex. $J$ Neurophysiol. 1998;79:1425-1440. doi:10.1152/ jn.1998.79.3.1425

16. Poellinger A. Olfactory habituation - a functional MRI study in humans. Futura, Boehringer Ingelheim Fonds. 1999.

17. Sobel N, Prabhakaran V, Zhao Z, et al. Time course of odorant-induced activation in the human primary olfactory cortex. J Neurophysiol. 2000;83:537-551. doi:10.1152/jn.2000.83.1.537

18. Poellinger A, Thomas R, Lio P, et al. Activation and habituation in olfaction-an fMRI study. NeuroImage. 2001;13:547-560. doi:10.1006/nimg.2000.0713

19. Dhilla Albers A, Asafu-Adjei J, Delaney MK, et al. Episodic memory of odors stratifies Alzheimer biomarkers in normal elderly. Ann Neurol. 2016;80:846-857. doi:10.1002/ana.24792

20. Jackson C, Rai N, McLean CK, et al. Overlapping risky decision-making and olfactory processing ability in HIV-infected individuals. Clin Exp Psychol. 2017;03. doi:10.4172/24712701.1000160

21. Nwulia EA, Rai N, Sartip K, et al. A pilot study of reduced olfactory bulb volume as a marker of PTSD in childhood trauma-exposed adult HIV-infected patients. J Trauma Stress. 2017;30:537-544. doi: $10.1002 /$ jts.22222
22. Bernstein JA, Hastings L, Boespflug EL, Allendorfer JB, Lamy M, Eliassen JC. Alteration of brain activation patterns in nonallergic rhinitis patients using functional magnetic resonance imaging before and after treatment with intranasal azelastine. Ann Allerg Asthma Immunol. 2011;106:527-532. doi:10.1016/j.anai.2011.02.014

23. Biswas SK, Vijayan K. Friction and wear of PTFE - a review. Wear. 1992;158:193-211. doi:10.1016/0043-1648(92)90039-B

24. Hummel T, Rissom K, Reden J, Hähner A, Weidenbecher M, Hüttenbrink K-B. Effects of olfactory training in patients with olfactory loss. Laryngoscope. 2009;119:496-499. doi:10.1002/lary.20101

25. Konstantinidis I, Tsakiropoulou E, Bekiaridou P, Kazantzidou C, Constantinidis J. Use of olfactory training in post-traumatic and postinfectious olfactory dysfunction. Laryngoscope. 2013;123:E85E90. doi:10.1002/lary.24390

26. Suzuki Y, Critchley HD, Suckling J, et al. Functional magnetic resonance imaging of odor identification: the effect of aging. J Gerontol a Biol Sci Med Sci. 2001;56:M756-M760. doi:10.1093/ gerona/56.12.M756

27. Kjelvik G, Evensmoen HR, Brezova V, Håberg AK. The human brain representation of odor identification. $J$ Neurophysiol. 2012;108:645-657. doi:10.1152/jn.01036.2010

28. Xiao W, Lv Q, Gao X, Sun Z, Yan X, Wei Y. Different brain activation in response to repeated odors of pleasantness and unpleasantness. Chemosens Percept. 2020;13:84-91. doi:10.1007/ s12078-019-09270-y

29. Kareken DA, Mosnik DM, Doty RL, Dzemidzic M, Hutchins GD. Functional anatomy of human odor sensation, discrimination, and identification in health and aging. Neuropsychology. 2003;17:482-495. doi:10.1037/0894-4105.17.3.482

30. Menon V, Uddin LQ. Saliency, switching, attention and control: a network model of insula function. Brain Struct Funct. 2010;214:655-667. doi:10.1007/s00429-010-0262-0

31. Levy LM, Henkin RI, Lin CS, Hutter A, Schellinger D. Odor memory induces brain activation as measured by functional MRI. $J$ Comput Assist Tomogr. 1999;23. doi:10.1097/00004728199907000-00001.

32. Wang J, Sun X, Yang QX. Early aging effect on the function of the human central olfactory system. J Gerontol a Biol Sci Med Sci. 2016; glw104. doi:10.1093/gerona/glw104

33. Shen J, Kassir MA, Wu J, et al. MR volumetric study of piriform-cortical amygdala and orbitofrontal cortices: the aging effect. PLoS One. 2013;8. doi:10.1371/journal.pone.0074526
Neuropsychiatric Disease and Treatment

\section{Publish your work in this journal}

Neuropsychiatric Disease and Treatment is an international, peerreviewed journal of clinical therapeutics and pharmacology focusing on concise rapid reporting of clinical or pre-clinical studies on a range of neuropsychiatric and neurological disorders. This journal is indexed on PubMed Central, the 'PsycINFO' database and CAS, and is the official journal of The International Neuropsychiatric Association (INA). The manuscript management system is completely online and includes a very quick and fair peer-review system, which is all easy to use. Visit http://www.dovepress.com/testimonials.php to read real quotes from published authors. 